\title{
CHEMICAL CLASS SPECIFICITY USING SELF-ASSEMBLED MONOLAYERS ON SAW DEVICES
}

\author{
Ross C. Thomas and Antonio J. Ricco \\ Microsensor R\&D Department, Sandia National Laboratories \\ Albuquerque, NM 87185-1425 \\ Huey C. Yang, Dan Dermody, and Richard M. Crooks \\ Department of Chemistry, Texas A\&M University \\ College Station, TX 77843-3255
}

\begin{abstract}
We have studied the chemical selectivity and sensitivity of surface acoustic wave (SAW) sensors covered by $\left(\mathrm{COO}^{-}\right)_{2} / \mathrm{Cu}^{2+}$ terminated interfaces by examining the response of selfassembled monolayer (SAM) films formed from the solution phase for 36,84 , and $180 \mathrm{~h}$ adsorption times. These SAMs were prepared on thin-film Au surfaces having variable, controlled grain size. Our results have important implications for designing and reliably fabricating chemical sensors that respond to specific organic analytes.
\end{abstract}

\section{INTRODUCTION}

Chemically sensitive SAW devices provide a platform for sensors that respond to specific organic analytes. ${ }^{1,2}$ Customdesigned and synthesized molecular recognition sites are an appealing means for obtaining chemical specificity, but the timeintensive nature of this approach, coupled with the plague of nonspecific adsorption and the large number of molecules for which sensors are sought, means that custom "guest-host" complexes may ultimately find application in a small fraction of successful chemical sensors. An alternate approach is to relax selectivity requirements significantly, using arrays of devices to provide a distinctive "fingerprint" pattem of responses for each analyte. ${ }^{3}$

We have designed chemically sensitive interfaces, of moderate class selectivity, that make use of known, reversible, bulk-phase interactions between functional groups in the film and a given analyte. ${ }^{4}$ These interfaces, which we immobilize on 97 . MHz ST-quartz SAW delay lines (Figure 1), are formed from composite SAM films employing organomercaptans. The rationale for using this kind of SAM is to provide a simple methodology to quickly and reliably fabricate chemically active vapor/solid interfaces that respond reversibly and reproducibly to a wide spectrum of organic analytes. For example, it is well documented that SAM films prepared using millimolar concentrations of $\boldsymbol{n}$-alkanethiols in ethanol produce monolayers having reproducible properties (i.e., surface coverage, structure, and orientation). ${ }^{5}$ Moreover, research has shown that the chemical reactivity of the vapor/solid interface is easily controlled by altering the SAM terminal groups. ${ }^{5}$

Organomercaptan SAM films are most often formed onto $\mathrm{Au}$ surfaces. However, the deposition method and the substrate materials used to produce the Au layer for attaching the SAM film commonly varies for different research groups. For example, Au films might be prepared by thermal, electron-beam, or sputter deposition. In many instances, a $\mathrm{Ti}, \mathrm{Cr}$, or organic adhesion layer is used to bond the $\mathrm{Au}$ to $\mathrm{Si}$, glass, quartz, or mica surfaces. Additional steps completed prior to forming the SAM film may include annealing or electropolishing the Au surface. Although

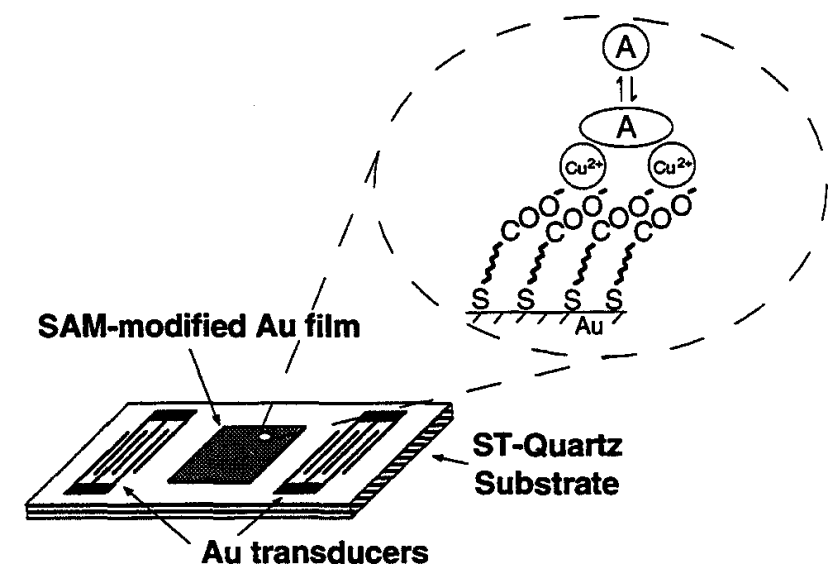

Figure 1. Schematic diagram of a self-assembled composite monolayer-modified chemically sensitive SAW device. The carboxylate coordinated $\mathrm{Cu}^{2+-t e r m i n a t e d ~ i n t e r f a c e ~ d e p i c t e d ~ h e r e ~}$ displays significant selectivity, in addition to reversibility and durability, for detecting organophosphorous compounds.

the wide range of substrate materials and deposition conditions produce Au films having different grain sizes and surface roughnesses, SAM films having similar properties are nevertheless formed. The ordering of the $S$ head group onto the primarily (111) crystallite orientation found to predominate for all of these Au surfaces, along with the densely-packed self assembly of the alkane portion of the organomercaptan molecules, most likely accounts for the consistent characteristics of the $\mathrm{Au}$ supported SAMs. ${ }^{5}$ Subtle effects (e.g., number of defects) within a SAM film, however, can result from differences in its microstructure, which is affected by the monolayer solution-phase adsorption time and/or the grain boundaries of the underlying $\mathrm{Au}$ film. ${ }^{6}$

In this paper, we compare the SAW response from a carboxylate coordinated $\mathrm{Cu}^{2+}$-terminated SAM to that from a methyl-terminated SAM, as these films interact with a vaporphase organophosphonate analyte as well as the vapors of a number of common organic solvents. (Selective detection of organophosphorous compounds, components of such substances as pesticides and chemical warfare agents, is important since these materials can pose a significant threat to health and environment.) We also examine in detail the effects of the solution-phase SAM adsorption time and the grain size of the supporting Au surface.

\section{EXPERIMENTAL METHODS}

The 97-MHz ST-quartz SAW devices were designed and fabricated at Sandia National Laboratories using interdigital transducers composed of fifty finger-pairs with a periodicity of 32 
$\mu \mathrm{m} .^{7}$ The transducers were defined photolithographically from $200 \mathrm{~nm}$-thick Au. A $15 \mathrm{~nm} \mathrm{Ti}$ adhesion layer was used to bond the $\mathrm{Au}$ to the quartz substrate. The composite SAMs were prepared on $100 \mathrm{~nm}$-thick Au films, which are electron-beam evaporated between the transducers. No $\mathrm{Cr}$ or $\mathrm{Ti}$ adhesion layer was used, since both of these metals are known to diffuse through $\mathrm{Au}$ films; this could interfere with comparisons between composite SAM films formed for different lengths of time and on $\mathrm{Au}$ surfaces having variable grain sizes. Au grain size was controlled by annealing the films at different temperatures.

Composite monolayer films were prepared on newly deposited Au films by soaking the substrates in a dilute solution of 11-mercaptoundecanoic acid (MUA) in ethanol for 36, 84, and $180 \mathrm{~h}$ followed by a $10 \mathrm{~min}$ solution-phase exposure to ethanolic $\mathrm{Cu}\left(\mathrm{ClO}_{4}\right)_{2} \cdot 6 \mathrm{H}_{2} \mathrm{O}$. Atomic force microscopy was used to determine the grain size of the SAM-modified $\mathrm{Au}$ film after completing the SAW measurements. A computer-controlled flow system was used to measure adsorption/desorption isotherms. ${ }^{8}$

The relationship between the frequency shift of a SAW delay-line oscillator circuit and the mass loading is described in detail elsewhere. ${ }^{9}$ Briefly, the change in frequency, $\Delta f$, is related to the change in adsorbed mass per area, $\Delta(m / A)$, when the SAW velocity is perturbed only by mass loading variations:

$$
\frac{\Delta f}{f_{\circ}}=-\kappa c_{m} f_{0} \times \Delta(m / A)
$$

where $c_{m}$ is the mass sensitivity $\left(1.33 \mathrm{~cm}^{2} / \mathrm{g}-\mathrm{MHz}\right.$ for ST-quartz), $f_{\mathrm{o}}$ is the unperturbed oscillator frequency $(97 \mathrm{MHz})$, and $\kappa$ is the fraction of the center-to-center distance between the transducers covered by the Au film; its present value is 0.7 .

\section{RESULTS AND DISCUSSION}

Figures $2 \mathrm{~A}$ and $2 \mathrm{~B}$ show adsorption isotherms as a function of vapor-phase concentration for a series of organic analytes, as well as water, interacting with a SAW device covered by either a $\left(\mathrm{COO}^{-}\right)_{2} / \mathrm{Cu}^{2+}$ - or a $\mathrm{CH}_{3}$-terminated monolayer film, respectively. For clarity, only a small percentage of the recorded data points are plotted. Since "raw" frequency changes are biased in favor of analytes with higher molecular weight, we determine the extent of selectivity by normalizing the data for analyte molecular weight, expressed as the number of moles adsorbed/area.

The adsorption isotherms in Figure 2A reveal preferential response to diisopropylmethylphosphonate (DIMP) and acetone relative to other common organic solvents. These two analytes are expected to have moderately strong interactions with the $\left(\mathrm{COO}^{-}\right)_{2} / \mathrm{Cu}^{2+}$-terminated surface because of their available oxygen lone pairs; the coordinated $\mathrm{Cu}^{2+}$ is a Lewis acid and these two analytes are Lewis bases. Only the DIMP analyte, however, shows an appreciable amount of specific binding with this composite monolayer film when the results from the methylterminated SAM are taken into account. The degree of specificity of adsorbate/SAM interactions is most readily ascertained by taking the differences between the isotherms of Figures $2 \mathrm{~A}$ and $2 \mathrm{~B}$, shown in Figure 2C. The data clearly indicate that the $\left(\mathrm{COO}^{-}\right)_{2} / \mathrm{Cu}^{2+}$-terminated SAM is almost 6 times more sensitive to DIMP than to acetone. The high level of specificity should apply to organophosphonates as a class.

The most important conclusion to be drawn from comparison of Figures $2 \mathrm{~A}$ and $2 \mathrm{~B}$ is that a pair of chemically sensitive SAW devices, one functionalized with the $\left(\mathrm{COO}^{-}\right)_{2} / \mathrm{Cu}^{2+}$ - and the other with the $\mathrm{CH}_{3}$-terminated monolayer film, readily distinguishes chemically specific from non-specific adsorption of analytes. For example, the similar responses for both acetone and water adsorbed on the two different SAM surfaces are implicative of interactions between the adsorbate molecules, not specific interactions with the SAM terminal groups. Water has a tendency to form H-bonded networks with itself, which probably explains the relatively large response. Since acetone has no $\mathrm{H}$-bonding protons, a similar effect would not be expected. However, the acetone was not rigorously dried, so the entrained vapor undoubtedly contains a small amount of water, which we suspect may participate in the formation of a $\mathrm{H}$ bonded Network.
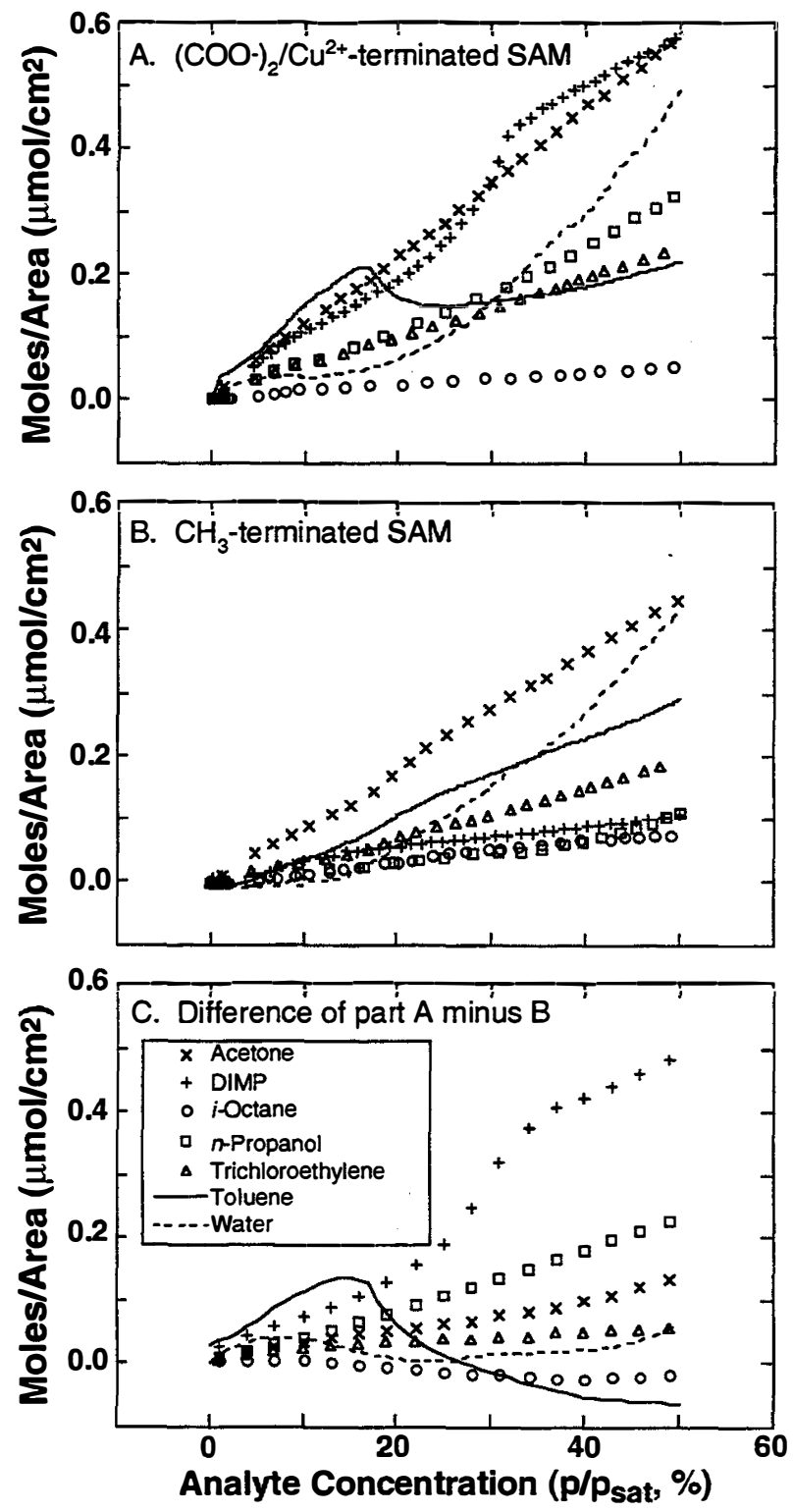

Figure 2. Adsorption isotherms for two SAW devices, covered with (A) (COO- $)_{2} / \mathrm{Cu}^{2+-}$ and (B) $\mathrm{CH}_{3}$-terminated monolayer films, exposed to six different organic analytes and water. (Responses are normalized for molecular weight). The degree of specificity of adsorbate-SAM interactions is most readily ascertained by comparing the difference isotherms obtained from part $(A)$ minus part $(B)$, shown in $(C)$. 


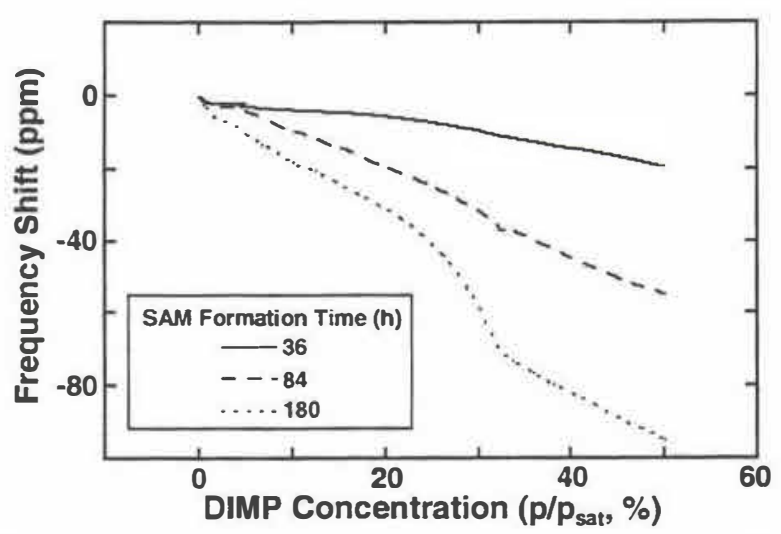

Figure 3. Adsorption isotherms of SAW devices covered by $(\mathrm{COO} \cdot)_{2} / \mathrm{Cu}^{2+-t e r m i n a t e d ~ i n t e r f a c e s ~ e x p o s e d ~ t o ~ D I M P . ~ T h e ~ M U A ~}$ $S A M s$ were prepared for 36,84 , and $180 \mathrm{~h}$. The frequency shift as a function of concentration increases with the SAM formation time. Enhanced sensitivity results from structural ordering effects at the vapor/solid interface of the composite monolayer film.

The responses measured for $n$-propanol and trichloroethylene further support our conclusion. The SAW device with the $\left(\mathrm{COO}^{-}\right)_{2} / \mathrm{Cu}^{2+}$-terminated SAM shows about three times the response for propanol as the methyl-terminated SAM, an indication of specific interaction between the propanol (and probably alcohols as a class) and the more polar monolayer film. For trichloroethylene, the response is only about $30 \%$ larger for the $\left(\mathrm{COO}^{-}\right)_{2} / \mathrm{Cu}^{2+}$-terminated film, indicating a minor role for specific interactions. Only two of the species examined actually show a larger surface coverage at $\mathrm{p} / \mathrm{p}_{\mathrm{sat}}=0.5$ for the methylterminated surface than for the $\left(\mathrm{COO}^{-}\right)_{2} / \mathrm{Cu}^{2+}$ surface: $i$-octane and toluene, the least polar of the analytes. In the case of toluene, the precise extent of the difference is clouded by the partially nonmass-related response observed in Figure 2A. For $i$-octane, though the response is small compared to the other analytes, it is nonetheless about $60 \%$ greater for the $\mathrm{CH}_{3}$-terminated surface, in accord with the very similar chemical nature of the surface and the analyte in this case.

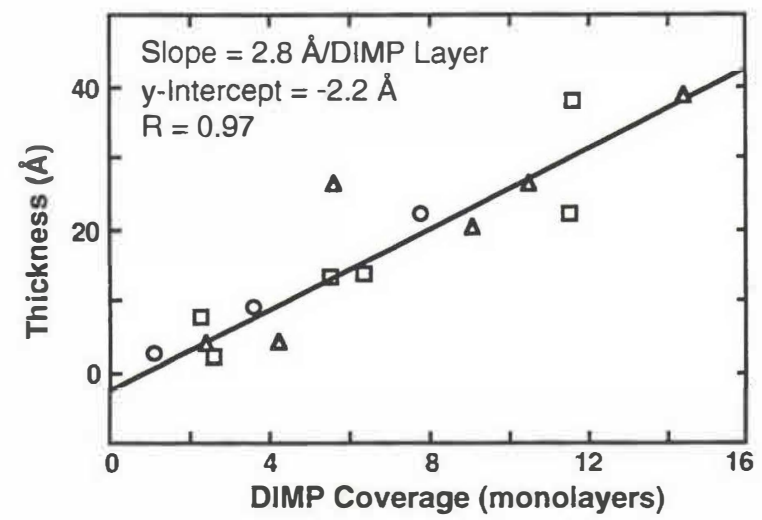

Figure 4. Ellipsometry data (y-axis) taken at the same time as SAW measurements (x-axis) for composite SAMs with MUA formation times of $36(0), 84(\square)$, and 180 ( $\Delta$ ) h. The DIMP vapor concentration was varied from $0-75 \%$ of saturation. The estimated coverage was determined from the frequency response using Eq. 1 and the bulk density of DIMP. The solid line is a linear fit thru all of the data. These results directly confirm multilayer adsorption of the DIMP analyte.
Figure 3 shows a series of DIMP adsorption isotherms obtained from SAW devices covered by $\left(\mathrm{COO}^{-}\right)_{2} / \mathrm{Cu}^{2+}$-terminated monolayer films that were assembled for 36,84 , and $180 \mathrm{~h}$ adsorption times. The binding affinity of this selective coating for DIMP increases dramatically as the MUA monolayer film is allowed to form for longer periods of time. Our results, which are consistent with DIMP multilayer formation (using Eq. 1), suggest that long adsorption times allow (re)organization of the SAM to provide a lower energy structure and an orientation of the $(\mathrm{COO})_{2} / \mathrm{Cu}^{2+}$-terminated layer that is more favorable for inducing ordering in adsorbed DIMP. The frequency shift for the 180-h film at $\mathrm{p} / \mathrm{p}_{\text {sat }}=50 \%$ is equivalent to 17 layers of adsorbed DIMP.

We have used in-situ ellipsometry combined with SAW measurements to provide detailed information about the multilayer adsorption response of the chemical sensor. The data indicate that the thickness of all the composite SAMs is $1.9 \pm 0.5$ $\mathrm{nm}$, which is in agreement with previous measurements and confirms that only a single monolayer of MUA forms (regardless of the solution-phase adsorption time). Figure 4 shows ellipsometry data ( $y$-axis) taken at the same time as SAW measurements (x-axis) for composite SAMs with MUA formation times of 36 ( ○ ), 84 ( $\mathbf{a}$ ), and 180 ( $\Delta$ ) h.. The DIMP vapor concentration was varied from $0-75 \%$ of saturation. We estimated the coverage from the frequency shift using Eq. 1 and the bulk density of DIMP.

As expected, the data show a linear relationship between the mass loading and film thickness (independent of the composite SAM film studied). The solid line represents a linear fit thru all

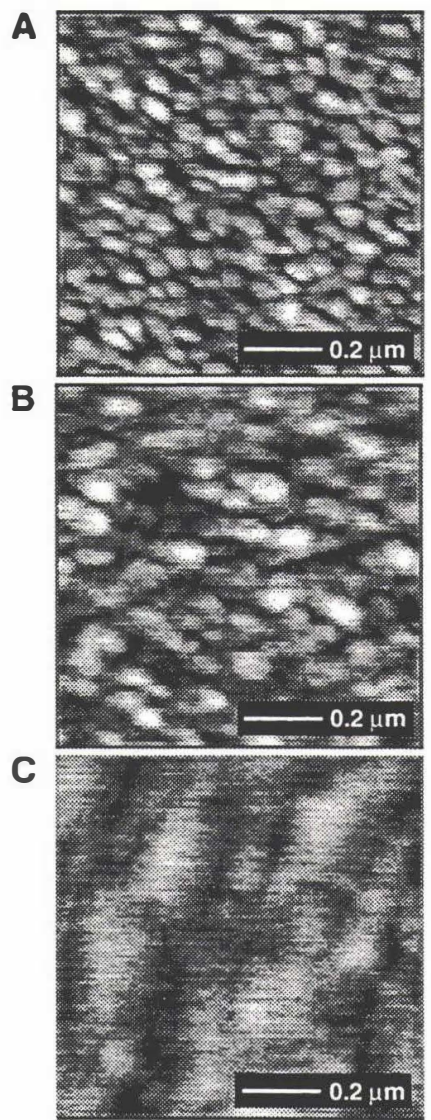

Figure 5. Constant repulsive-force images of $100 \mathrm{~nm}$-thick Au films evaporated onto ST-quant SAW devices. The average grain size, which we controlled by annealing the devices at different temperatures, was (A) $50 \mathrm{~nm}$, (B) $80 \mathrm{~nm}$, and (C) $240 \mathrm{~nm}$. 


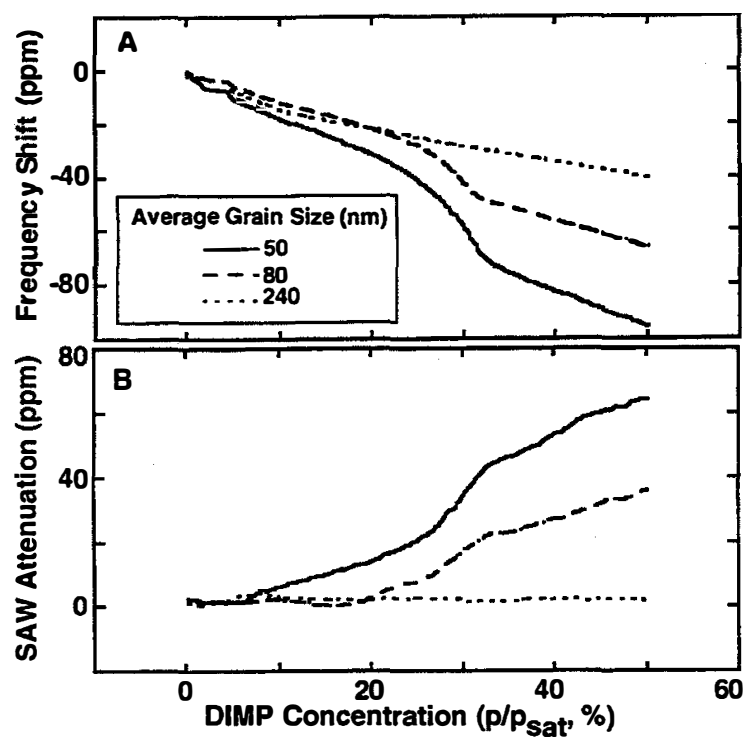

Figure 6. DIMP adsorption isotherms for composite monolayer films prepared on the Au surfaces having the variable grain sizes shown in Figure 5. (A) Frequency shift and (B) SAW attenuation versus the vapor-phase concentration of DIMP illustrate that sensitivity of SAW devices with composite SAMs prepared on $A u$ films increases as grain size decreases.

of the data with a slope of $2.8 \AA$ NIMP layer and a y-intercept of $-2.2 \AA$. Because each DIMP layer should be about $6.0 \AA$ thick, the results suggest that the SAW measurements overestimate the mass loading by a factor of 2 . We have not, however, taken into account non-specific adsorption of the DIMP analyte over the active region of the SAW device not covered by Au. Control experiments using bare quartz SAW devices exposed to DIMP show a measurable response that is partly irreversible, irreproducible, and dependent on surface cleanliness. Thus, the data do confirm multilayer adsorption, but additional measurements are necessary to reconcile quantitatively the SAW and ellipsometry responses.

The composite-SAM-modified $\mathrm{Au}$ surfaces shown in Figures 5A, 5B, and 5C produced the DIMP isotherm data shown in Figure 6 by the solid, dashed, and dotted lines, respectively. Clearly, as the grain size of the Au film decreases (by a factor of about 5), there is a significant increase in the extent of adsorption of DIMP at a given $\mathrm{p} / \mathrm{p}_{\text {sat }}$. We used electrochemical measurements to verify that surface roughness is not the dominant factor in these results: while the roughness changes by $11 \%$, the SAW response varies by a factor of 2.5 at $\mathrm{p} / \mathrm{p}_{\text {sat }}=0.5$ from the largest to smallest grain size. Since the crystallite orientation of the $\mathrm{Au}$ is predominantly (111) and is not altered by annealing, the total coverage of the MUA SAMs for the three grain sizes should be very similar. The attenuation of the acoustic wave (Figure 6B) indicates that the grain size also has a strong influence on the structural properties of the SAM and its consequent interactions with adsorbed analyte.

\section{SUMMARY AND CONCLUSIONS}

Our results illustrate that a pair of SAM-functionalized SAW sensors, one bearing a methyl- and the other a $\left(\mathrm{COO}^{-}\right)_{2} / \mathrm{Cu}^{2+}$-terminated monolayer, allow clear distinction to be drawn between chemically specific and nonspecific adsorption of analytes. The results further show that the composite SAM films
(1) preferentially adsorb particular classes of organic analytes in a manner that follows from simple concepts such as Lewis acid/base, H-bonding, and polar vs. nonpolar interactions, (2) adsorb a greater quantity of DIMP at a given partial pressure for longer solution-phase formation times of the organomercaptan monolayer, and (3) adsorb a greater quantity of DIMP at a given partial pressure as the grain size of the supporting Au film decreases. While result (1) is straightforward and to be expected, we believe results (2) and (3) are consequences of the extent and nature of the ordering of the SAM and its outer surface: the ordering affects the thermodynamics of adsorption of multilayers. The results from these measurements are an important step toward reliably fabricating chemical sensor arrays that respond to organic analytes with controlled class and/or molecular specificity.

We gratefully acknowledge the excellent technical assistance of $\mathrm{Al}$ Staton and Mary-Anne Mitchell of Sandia National Laboratories. This work was supported by the U.S. DOE under contract DE-ACO4-94AL85000 and by the National Science Foundation (CHE-9312441).

\section{REFERENCES}

1. R. C. Hughes, A. J. Ricco, M. A. Butler, and S. J. Martin, "Chemical Microsensors", Science, 254, 74 (1991).

2. J. W. Grate, S. J. Martin, and R. M. White, "Acoustic Wave Microsensors", Anal. Chem., 65, 940A (1993).

3. A. J. Ricco, G. C. Osbourn, J. W. Bartholomew, R. M. Crooks, C. Xu, and R. E. Allred, "New Materials and Multidimensional Cluster Analysis for SAW Chemical Sensor Arrays", Technical Digest of the 1994 Solid-State Sensor and Actuator Workshop, Hilton Head Isl., SC, 6/13-16/94 Transducer Research Foundation, Cleveland (1994), pp. 180-183.

4. R. C. Thomas, H. C. Yang, C. R. DiRubio, A. J. Ricco, and R. M. Crooks, "Chemically Sensitive Surface Acoustic Wave Devices Employing a Self-Assembled Composite Monolayer Film: Molecular Specificity and Effects Due to SAM Adsorption Time and Au Surface Morphology," Langmuir, accepted for publication (12/95).

5. L. H. Dubois and R. G. Nuzzo, "Synthesis, Structure, and Properties of Model Organic Surfaces", Annu. Rev. Phys. Chem., 43, 437 (1992) and references therein.

6. G. E. Poirier and M. J. Tarlov, "Molecular Ordering and Gold Migration Observed in Butanethiol Self-Assembled Monolayers", J. Phys. Chem. ,99, 10966, (1995).

7. A. J. Ricco and S. J. Martin, "Thin metal film characterization and chemical sensors: monitoring electronic conductivity, mass loading and mechanical properties with surface acoustic wave devices", Thin Solid Films, 206, 94 (1991).

8. N. W. Ashcroft and N. D. Mermin, Solid State Physics; Saunders College (1976), Chapter 4, p. 63.

9. S. J. Martin, A. J. Ricco, D. S. Ginley, and T. E. Zipperian, "Isothermal Measurements and Thermal Desorption of Organic Vapors Using SAW Devices", IEEE Trans. on UFFC, UFFC-34, $142(1987)$ 